\title{
Controllability and Accumulation of Errors Arising in a General Iteration Method
}

\author{
Faik Gürsoy $^{1 *}$, Abdul Rahim Khan ${ }^{2}$ and Kadri Doğan ${ }^{3}$ \\ ${ }^{1}$ Department of Mathematics, Adiyaman University, Adiyaman 02040, Turkey \\ ${ }^{2}$ Department of Mathematics and Statistics, King Fahd University of Petroleum and Minerals, Dhahran 31261, Saudi Arabia \\ ${ }^{3}$ Department of Computer Engineering, Artvin Coruh University, 08000, Artvin, Turkey \\ * Corresponding author
}

\author{
Article Info \\ Keywords: Error analysis, Random er- \\ rors, $T S$ iteration method \\ 2010 AMS: 47H10, 97N20, 47J25, \\ $54 \mathrm{H} 25$ \\ Received: 18 October 2020 \\ Accepted: 24 May 2021 \\ Available online: 27 May 2021
}

\begin{abstract}
In this paper, we propose and analyze a three-step general iteration method which is a special case of an iteration method proposed in (S. Thianwan and S. Suantai, Convergence criteria of a new three-step iteration with errors for nonexpansive nonself-mappings, Comput. Math. Appl. 52 (2006), 1107-1118). Here we intend to study directly the accumulation, estimation and control of random errors in the newly proposed general iteration method. We give conditions under which the accumulated-error in our iteration method is bounded and controllable in a permissible range.
\end{abstract}

\section{Introduction}

The tools of fixed point theory are successfully applied to the solutions of a wide variety of problems arising in many disciplines of science. In particular, fixed point iteration methods have attracted the attention of researchers and in parallel with the extension of the application areas of fixed point theory, a great deal of effort has been devoted to the study of some important features of iteration methods (see, for instance, [1]-[9]).

Errors usually occur in the iterative calculations and so consideration of error estimates is of utmost importance in the study of iteration methods. A quick look at literature reveals that many paper have been devoted to the study of iteration methods with errors where the errors are calculated indirectly. There are only a few papers concerning direct estimation and control of errors of the iteration methods (see, e.g., [10]-[12]).

Throughout this exposition, we assume that $(B,\|\cdot\|)$ is an arbitrary real Banach space, $S$ a nonempty closed and convex subset of $B, T: S \rightarrow S$ an operator, and $\left\{a_{n}\right\}_{n=0}^{\infty},\left\{b_{n}\right\}_{n=0}^{\infty},\left\{c_{n}\right\}_{n=0}^{\infty},\left\{\alpha_{n}\right\}_{n=0}^{\infty},\left\{\beta_{n}\right\}_{n=0}^{\infty},\left\{\lambda_{n}\right\}_{n=0}^{\infty},\left\{\mu_{n}\right\}_{n=0}^{\infty},\left\{\gamma_{n}\right\}_{n=0}^{\infty},\left\{\alpha_{n}+\beta_{n}+\lambda_{n}\right\}_{n=0}^{\infty}$, $\left\{b_{n}+c_{n}+\mu_{n}\right\}_{n=0}^{\infty},\left\{a_{n}+\gamma_{n}\right\}_{n=0}^{\infty} \subseteq[0,1]$ are parameter sequences satisfying certain control condition(s) and $\left\{u_{n}\right\}_{n=0}^{\infty},\left\{v_{n}\right\}_{n=0}^{\infty}$, $\left\{w_{n}\right\}_{n=0}^{\infty}$ are bounded sequences in $S$.

In 2006, Thianwan and Suantai [13] defined a three-step iteration method on $S$ with error terms as:

$$
\left\{\begin{array}{c}
x_{0} \in S, \\
x_{n+1}=\left(1-\alpha_{n}-\beta_{n}-\lambda_{n}\right) x_{n}+\alpha_{n} T y_{n}+\beta_{n} T z_{n}+\lambda_{n} w_{n} \\
y_{n}=\left(1-b_{n}-c_{n}-\mu_{n}\right) x_{n}+b_{n} T z_{n}+c_{n} T x_{n}+\mu_{n} v_{n} \\
z_{n}=\left(1-a_{n}-\gamma_{n}\right) x_{n}+a_{n} T x_{n}+\gamma_{n} u_{n}, \text { for all } n \in \mathbb{N} .
\end{array}\right.
$$

The iteration method (1.1) has been used for approximation of fixed points of various nonlinear mappings (see, for instance, 
$[14,15])$. If we put $\lambda_{n}=\mu_{n}=\gamma_{n}=0$ for all $n \in \mathbb{N}$ in (1.1), then we obtain

$$
\left\{\begin{array}{c}
x_{0} \in S, \\
x_{n+1}=\left(1-\alpha_{n}-\beta_{n}\right) x_{n}+\alpha_{n} T y_{n}+\beta_{n} T z_{n} \\
y_{n}=\left(1-b_{n}-c_{n}\right) x_{n}+b_{n} T z_{n}+c_{n} T x_{n} \\
z_{n}=\left(1-a_{n}\right) x_{n}+a_{n} T x_{n}, \text { for all } n \in \mathbb{N} .
\end{array}\right.
$$

Remark 1.1. The iteration method (1.1) reduces to:

(i) Noor iteration method [16] if $c_{n}=\beta_{n}=\gamma_{n}=\lambda_{n}=\mu_{n}=0$ for all $n \in \mathbb{N}$,

(ii) Ishikawa iteration method [17] if $a_{n}=c_{n}=\beta_{n}=\gamma_{n}=\lambda_{n}=\mu_{n}=0$ for all $n \in \mathbb{N}$,

(iii) Mann iteration method [18] if $a_{n}=b_{n}=c_{n}=\beta_{n}=\gamma_{n}=\lambda_{n}=\mu_{n}=0$ for all $n \in \mathbb{N}$.

\section{Main results}

Here we intend to study directly the accumulation, estimation and control of random errors in the iteration method (1.2). Define the errors of $T x_{n}, T y_{n}$ and $T z_{n}$ by

$$
u_{n}=T x_{n}-\overline{T x_{n}}, v_{n}=T z_{n}-\overline{T z_{n}} \text { and } w_{n}=T y_{n}-\overline{T y_{n}}
$$

for all $n \in \mathbb{N}$, where $\overline{T x_{n}}, \overline{T y_{n}}$ and $\overline{T z_{n}}$ are the exact values of $T x_{n}, T y_{n}$ and $T z_{n}$ respectively, that is, $T x_{n}, T y_{n}$ and $T z_{n}$ are approximate values of $\overline{T x_{n}}, \overline{T y_{n}}$ and $\overline{T z_{n}}$, respectively. The theory of errors implies that $\left\{u_{n}\right\}_{n=0}^{\infty},\left\{v_{n}\right\}_{n=0}^{\infty}$ and $\left\{w_{n}\right\}_{n=0}^{\infty}$ are bounded. Set

$$
B=\max \left\{B_{u}, B_{v}, B_{w}\right\}
$$

where $B_{u}=\sup _{n \in \mathbb{N}}\left\|u_{n}\right\|, B_{v}=\sup _{n \in \mathbb{N}}\left\|v_{n}\right\|$ and $B_{w}=\sup _{n \in \mathbb{N}}\left\|w_{n}\right\|$ are the bounds on the absolute errors of $\left\{T x_{n}\right\}_{n=0}^{\infty},\left\{T z_{n}\right\}_{n=0}^{\infty}$ and $\left\{T y_{n}\right\}_{n=0}^{\infty}$, respectively.

The main part of accumulation of errors from (1.2) comes essentially from $u_{n}, v_{n}$ and $w_{n}$; hence we can set

$$
\left\{\begin{array}{c}
\overline{x_{0}} \in S, \\
\overline{x_{n+1}}=\left(1-\alpha_{n}-\beta_{n}\right) \overline{x_{n}}+\alpha_{n} \overline{T y_{n}}+\beta_{n} \overline{T z_{n}} \\
\overline{y_{n}}=\left(1-b_{n}-c_{n}\right) \overline{x_{n}}+b_{n} \overline{T z_{n}}+c_{n} \overline{T x_{n}} \\
\overline{z_{n}}=\left(1-a_{n}\right) \overline{x_{n}}+a_{n} \overline{T x_{n}}, \text { for all } n \in \mathbb{N} .
\end{array}\right.
$$

where $\overline{x_{n}}, \overline{y_{n}}$ and $\overline{z_{n}}$ are exact values of $x_{n}, y_{n}$ and $z_{n}$, respectively. Clearly, the errors of last iteration will affect the next $(n+1)$ steps. So, utilizing (1.2), (2.1) and (2.3), we have

$$
\begin{aligned}
x_{0}= & \overline{x_{0}} \\
z_{0}= & \left(1-a_{0}\right) x_{0}+a_{0} T x_{0} \\
= & \left(1-a_{0}\right) \overline{x_{0}}+a_{0} \overline{T x_{0}}+a_{0} u_{0}=\overline{z_{0}}+a_{0} u_{0} ; \\
y_{0}= & \left(1-b_{0}-c_{0}\right) x_{0}+b_{0} T z_{0}+c_{0} T x_{0} \\
= & \left(1-b_{0}-c_{0}\right) \overline{x_{0}}+b_{0} \overline{T z_{0}}+c_{0} \overline{T x_{0}}+b_{0} v_{0}+c_{0} u_{0} \\
= & \overline{y_{0}}+b_{0} v_{0}+c_{0} u_{0} ; \\
x_{1}= & \left(1-\alpha_{0}-\beta_{0}\right) x_{0}+\alpha_{0} T y_{0}+\beta_{0} T z_{0} \\
= & \left(1-\alpha_{0}-\beta_{0}\right) \overline{x_{0}}+\alpha_{0} \overline{T y_{0}}+\beta_{0} \overline{T z_{0}}+\alpha_{0} w_{0}+\beta_{0} v_{0} \\
= & \overline{x_{1}}+\alpha_{0} w_{0}+\beta_{0} v_{0} ; \\
z_{1}= & \overline{z_{1}}+\left(1-a_{1}\right)\left(\alpha_{0} w_{0}+\beta_{0} v_{0}\right)+a_{1} u_{1} ; \\
y_{1}= & \overline{y_{1}}+\left(1-b_{1}-c_{1}\right)\left(\alpha_{0} w_{0}+\beta_{0} v_{0}\right)+b_{1} v_{1}+c_{1} u_{1} ; \\
x_{2}= & \overline{x_{2}}+\left(1-\alpha_{1}-\beta_{1}\right)\left(\alpha_{0} w_{0}+\beta_{0} v_{0}\right)+\alpha_{1} w_{1}+\beta_{1} v_{1} ; \\
& \\
z_{2}= & \overline{z_{2}}+\left(1-a_{2}\right)\left(1-\alpha_{1}-\beta_{1}\right)\left(\alpha_{0} w_{0}+\beta_{0} v_{0}\right) \\
& +\left(1-a_{2}\right)\left(\alpha_{1} w_{1}+\beta_{1} v_{1}\right)+a_{2} u_{2} ; \\
y_{2}= & \overline{y_{2}}+\left(1-b_{2}-c_{2}\right)\left[\left(1-\alpha_{1}-\beta_{1}\right)\left(\alpha_{0} w_{0}+\beta_{0} v_{0}\right)\right. \\
& \left.+\left(\alpha_{1} w_{1}+\beta_{1} v_{1}\right)\right]+b_{2} v_{2}+c_{2} u_{2} ; \\
x_{3}= & \overline{x_{3}}+\left(1-\alpha_{2}-\beta_{2}\right)\left(1-\alpha_{1}-\beta_{1}\right)\left(\alpha_{0} w_{0}+\beta_{0} v_{0}\right) \\
& +\left(1-\alpha_{2}\right)\left(\alpha_{1} w_{1}+\beta_{1} v_{1}\right)+\alpha_{2} w_{2}+\beta_{2} v_{2} ;
\end{aligned}
$$


Repeating the above process, we obtain

$$
\begin{gathered}
x_{n+1}=\overline{x_{n+1}}+\sum_{k=0}^{n}\left(\alpha_{k} w_{k}+\beta_{k} v_{k}\right)\left[\prod_{i=k+1}^{n}\left(1-\alpha_{i}-\beta_{i}\right)\right], \\
y_{n}=\overline{y_{n}}+b_{n} v_{n}+c_{n} u_{n}+\left(1-b_{n}-c_{n}\right) \sum_{k=0}^{n-1}\left(\alpha_{k} w_{k}+\beta_{k} v_{k}\right)\left[\prod_{i=k+1}^{n-1}\left(1-\alpha_{i}-\beta_{i}\right)\right] \\
=\overline{y_{n}}+b_{n} v_{n}+c_{n} u_{n}+\left(1-b_{n}-c_{n}\right)\left(x_{n}-\overline{x_{n}}\right),
\end{gathered}
$$

and

$$
\begin{aligned}
z_{n} & =\overline{z_{n}}+a_{n} u_{n}+\left(1-a_{n}\right) \sum_{k=0}^{n-1}\left(\alpha_{k} w_{k}+\beta_{k} v_{k}\right)\left[\prod_{i=k+1}^{n-1}\left(1-\alpha_{i}-\beta_{i}\right)\right] \\
& =\overline{z_{n}}+a_{n} u_{n}+\left(1-a_{n}\right)\left(x_{n}-\overline{x_{n}}\right) \text { for all } n \in \mathbb{N} .
\end{aligned}
$$

Define

$$
\begin{gathered}
Q_{n}^{(1)}:=x_{n+1}-\overline{x_{n+1}}=\sum_{k=0}^{n}\left(\alpha_{k} w_{k}+\beta_{k} v_{k}\right)\left[\prod_{i=k+1}^{n}\left(1-\alpha_{i}-\beta_{i}\right)\right] \\
Q_{n}^{(2)}:=y_{n}-\overline{y_{n}}=b_{n} v_{n}+c_{n} u_{n}+\left(1-b_{n}-c_{n}\right) Q_{n-1}^{(1)},
\end{gathered}
$$

and

$$
Q_{n}^{(3)}:=z_{n}-\overline{z_{n}}=a_{n} u_{n}+\left(1-a_{n}\right) Q_{n-1}^{(1)} \text { for all } n \in \mathbb{N}
$$

Obviously, the errors of iteration method, after $(n+1)$ times iterations, are added up to $Q_{n}^{(1)}, Q_{n}^{(2)}$ and $Q_{n}^{(3)}$. Now, we are in a position to give the following result.

Theorem 2.1. Let $S, T, B, Q_{n}^{(1)}, Q_{n}^{(2)}$ and $Q_{n}^{(3)}$ be as above.

(i) If $\sum_{i=0}^{\infty}\left(\alpha_{i}+\beta_{i}\right)=+\infty$, then the accumulation of errors in (1.2) is bounded and does not exceed the number B;

(ii) If $\sum_{i=0}^{\infty}\left(\alpha_{i}+\beta_{i}\right)<+\infty, \lim _{n \rightarrow \infty} a_{n}=0$ and $\lim _{n \rightarrow \infty}\left(b_{n}+c_{n}\right)=0$, then random errors of (1.2) are controllable.

Proof. (i) It is well known that $\sum_{i=0}^{\infty}\left(\alpha_{i}+\beta_{i}\right)=+\infty$ implies $\prod_{i=0}^{\infty}\left(1-\alpha_{i}-\beta_{i}\right)=0$ (see, e.g., (Remark 2.1 of [19])). From (2.2), 
(2.4)-(2.6) we have

$$
\begin{aligned}
& \left\|Q_{n}^{(1)}\right\|=\|\left(\alpha_{0} w_{0}+\beta_{0} v_{0}\right) \prod_{i=1}^{n}\left(1-\alpha_{i}-\beta_{i}\right) \\
& +\left(\alpha_{1} w_{1}+\beta_{1} v_{1}\right) \prod_{i=2}^{n}\left(1-\alpha_{i}-\beta_{i}\right) \\
& +\cdots+\left(\alpha_{n-1} w_{n-1}+\beta_{n-1} v_{n-1}\right) \prod_{i=n}^{n}\left(1-\alpha_{i}-\beta_{i}\right)+\alpha_{n} w_{n}+\beta_{n} v_{n} \| \\
& \leq\left\|\left(\alpha_{0} w_{0}+\beta_{0} v_{0}\right) \prod_{i=1}^{n}\left(1-\alpha_{i}-\beta_{i}\right)\right\| \\
& +\left\|\left(\alpha_{1} w_{1}+\beta_{1} v_{1}\right) \prod_{i=2}^{n}\left(1-\alpha_{i}-\beta_{i}\right)\right\| \\
& +\cdots+\left\|\left(\alpha_{n-1} w_{n-1}+\beta_{n-1} v_{n-1}\right) \prod_{i=n}^{n}\left(1-\alpha_{i}-\beta_{i}\right)\right\| \\
& +\left\|\alpha_{n} w_{n}+\beta_{n} v_{n}\right\| \\
& \leq\left(\alpha_{0}\left\|w_{0}\right\|+\beta_{0}\left\|v_{0}\right\|\right) \prod_{i=1}^{n}\left(1-\alpha_{i}-\beta_{i}\right) \\
& +\left(\alpha_{1}\left\|w_{1}\right\|+\beta_{1}\left\|v_{1}\right\|\right) \prod_{i=2}^{n}\left(1-\alpha_{i}-\beta_{i}\right) \\
& +\cdots+\left(\alpha_{n-1}\left\|w_{n-1}\right\|+\beta_{n-1}\left\|v_{n-1}\right\|\right) \prod_{i=n}^{n}\left(1-\alpha_{i}-\beta_{i}\right) \\
& +\alpha_{n}\left\|w_{n}\right\|+\beta_{n}\left\|v_{n}\right\| \\
& \leq B\left\{\left(\alpha_{0}+\beta_{0}\right) \prod_{i=1}^{n}\left(1-\alpha_{i}-\beta_{i}\right)+\left(\alpha_{1}+\beta_{1}\right) \prod_{i=2}^{n}\left(1-\alpha_{i}-\beta_{i}\right)\right. \\
& \left.+\cdots+\left(\alpha_{n-1}+\beta_{n-1}\right) \prod_{i=n}^{n}\left(1-\alpha_{i}-\beta_{i}\right)+\alpha_{n}+\beta_{n}\right\} \\
& =B\left\{\prod_{i=0}^{n}\left(1-\alpha_{i}-\beta_{i}\right)+\left(\alpha_{0}+\beta_{0}\right) \prod_{i=1}^{n}\left(1-\alpha_{i}-\beta_{i}\right)\right. \\
& +\left(\alpha_{1}+\beta_{1}\right) \prod_{i=2}^{n}\left(1-\alpha_{i}-\beta_{i}\right)+\cdots+\left(\alpha_{n-1}+\beta_{n-1}\right) \prod_{i=n}^{n}\left(1-\alpha_{i}-\beta_{i}\right) \\
& \left.+\alpha_{n}+\beta_{n}-\prod_{i=0}^{n}\left(1-\alpha_{i}-\beta_{i}\right)\right\} \\
& =B\left[1-\prod_{i=0}^{n}\left(1-\alpha_{i}-\beta_{i}\right)\right] \leq B\left[1-\prod_{i=0}^{\infty}\left(1-\alpha_{i}-\beta_{i}\right)\right]=B \text {, } \\
& \left\|Q_{n}^{(2)}\right\|=\left\|b_{n} v_{n}+c_{n} u_{n}+\left(1-b_{n}-c_{n}\right) Q_{n-1}^{(1)}\right\| \\
& \leq b_{n}\left\|v_{n}\right\|+c_{n}\left\|u_{n}\right\|+\left(1-b_{n}-c_{n}\right)\left\|Q_{n-1}^{(1)}\right\| \\
& \leq B\left(b_{n}+c_{n}\right)+\left(1-b_{n}-c_{n}\right) B=B
\end{aligned}
$$

and

$$
\begin{aligned}
\left\|Q_{n}^{(3)}\right\| & =\left\|a_{n} u_{n}+\left(1-a_{n}\right) Q_{n-1}^{(1)}\right\| \\
& \leq a_{n}\left\|u_{n}\right\|+\left(1-a_{n}\right)\left\|Q_{n-1}^{(1)}\right\| \\
& \leq a_{n} B+\left(1-a_{n}\right) B=B \text { for all } n \in \mathbb{N} .
\end{aligned}
$$

Hence, we have $\max _{n \in \mathbb{N}}\left\{\left\|Q_{n}^{(1)}\right\|,\left\|Q_{n}^{(2)}\right\|,\left\|Q_{n}^{(3)}\right\|\right\} \leq B$. 
(ii) Indeed, $\sum_{i=0}^{\infty}\left(\alpha_{i}+\beta_{i}\right)<+\infty$ implies that $\prod_{i=0}^{\infty}\left(1-\alpha_{i}-\beta_{i}\right) \in(0,1)$. Let $1-\prod_{i=0}^{\infty}\left(1-\alpha_{i}-\beta_{i}\right)=\ell \in(0,1)$. Thus, from (2.7), we obtain

$$
\left\|Q_{n}^{(1)}\right\| \leq B\left[1-\prod_{i=0}^{\infty}\left(1-\alpha_{i}-\beta_{i}\right)\right] \leq \ell B \text { for all } n \in \mathbb{N} .
$$

On the other hand, the condition $\lim _{n \rightarrow \infty}\left(b_{n}+c_{n}\right)=0$ implies the existence of an $n_{0} \in \mathbb{N}$ such that for all $n \geq n_{0}$ we have $b_{n}+c_{n} \leq \ell /(1-\ell)$. Using this fact together with (2.8) and (2.10), we get

$$
\begin{aligned}
\left\|Q_{n}^{(2)}\right\| & \leq\left(b_{n}+c_{n}\right) B+\left(1-b_{n}-c_{n}\right)\left\|Q_{n-1}^{(1)}\right\| \\
& \leq\left(b_{n}+c_{n}\right) B(1-\ell)+B \ell \\
& \leq \frac{\ell}{1-\ell} B(1-\ell)+B \ell=2 B \ell \text { for all } n \geq n_{0} .
\end{aligned}
$$

Similarly, the condition $\lim _{n \rightarrow \infty} a_{n}=0$ implies the existence of an $n_{0} \in \mathbb{N}$ such that for all $n \geq n_{0}$ we have $a_{n} \leq \ell /(1-\ell)$. Hence, from (2.9) and (2.10), we have

$$
\begin{aligned}
\left\|Q_{n}^{(3)}\right\| & \leq a_{n}\left\|u_{n}\right\|+\left(1-a_{n}\right)\left\|Q_{n-1}^{(1)}\right\| \\
& \leq a_{n} B(1-\ell)+B \ell \\
& \leq \frac{\ell}{1-\ell} B(1-\ell)+B \ell=2 B \ell \text { for all } n \geq n_{0} .
\end{aligned}
$$

Thus, we conclude that $\left\|Q_{n}^{(1)}\right\|,\left\|Q_{n}^{(2)}\right\|$ and $\left\|Q_{n}^{(3)}\right\|$ can be controlled for suitable choice of the parameter sequences $\left\{a_{n}\right\}_{n=0}^{\infty}$, $\left\{b_{n}\right\}_{n=0}^{\infty},\left\{c_{n}\right\}_{n=0}^{\infty},\left\{\alpha_{n}\right\}_{n=0}^{\infty}$ and $\left\{\beta_{n}\right\}_{n=0}^{\infty}$ for all $n \geq n_{0}$.

Example 2.2. Let $\alpha_{n}+\beta_{n}=\frac{1}{\left(n^{2}+4 n+3\right)^{2}}$ for all $n \in \mathbb{N}$. Then, we have by the Wolfram Mathematica 9 software package that $\sum_{i=0}^{\infty}\left(\alpha_{i}+\beta_{i}\right)=\frac{1}{48}\left(4 \pi^{2}-33\right)<+\infty$ and $\ell=1-\prod_{i=0}^{\infty}\left(1-\alpha_{i}-\beta_{i}\right)=1+\frac{2 \sqrt{2} \sin (\sqrt{2} \pi)}{\pi} \approx 0.132183 \in(0,1)$ which implies together with (2.10)-(2.12) that $\left\|Q_{n}^{(1)}\right\| \leq\left(1+\frac{2 \sqrt{2} \sin (\sqrt{2} \pi)}{\pi}\right) B,\left\|Q_{n}^{(2)}\right\| \leq 2\left(1+\frac{2 \sqrt{2} \sin (\sqrt{2} \pi)}{\pi}\right) B$ and $\left\|Q_{n}^{(3)}\right\| \leq 2\left(1+\frac{2 \sqrt{2} \sin (\sqrt{2} \pi)}{\pi}\right) B$ for all $n \in \mathbb{N}$.

Especially, for any $\varepsilon \in(0,1)$, if $\alpha_{n}+\beta_{n}=\frac{5^{n+2}}{7^{n+3}} \varepsilon$ for all $n \in \mathbb{N}$, then

$$
\prod_{i=0}^{\infty}\left(1-\alpha_{i}-\beta_{i}\right) \geq 1-\sum_{i=0}^{\infty}\left(\alpha_{i}+\beta_{i}\right)=1-\frac{25}{98} \varepsilon
$$

which yields $\ell<\frac{25}{98} \varepsilon$, so that

$$
\begin{gathered}
\left\|Q_{n}^{(1)}\right\| \leq \frac{25}{98} \varepsilon B \text { for all } n \in \mathbb{N}, \\
\left\|Q_{n}^{(2)}\right\| \leq \frac{25}{49} \varepsilon B \text { for all } n \geq n_{0},
\end{gathered}
$$

and

$$
\left\|Q_{n}^{(3)}\right\| \leq \frac{25}{49} \varepsilon B \text { for all } n \geq n_{0},
$$

where $n_{0}$ belongs to $\mathbb{N}$ and the inequalities $a_{n} \leq \frac{\varepsilon}{3.92-\varepsilon}$ and $b_{n}+c_{n} \leq \frac{\varepsilon}{3.92-\varepsilon}$ hold. Hence, the random errors is controllable in a permissible range for suitable choice of the parameter sequences $\left\{a_{n}\right\}_{n=0}^{\infty},\left\{b_{n}\right\}_{n=0}^{\infty},\left\{c_{n}\right\}_{n=0}^{\infty},\left\{\alpha_{n}\right\}_{n=0}^{\infty}$ and $\left\{\beta_{n}\right\}_{n=0}^{\infty}$ for all $n \geq n_{0}$.

\section{Acknowledgements}

The authors would like to express their sincere thanks to the editor and the anonymous reviewers for their helpful comments and suggestions.

\section{Funding}

There is no funding for this work. 


\section{Availability of data and materials}

Not applicable.

\section{Competing interests}

The authors declare that they have no competing interests.

\section{Author's contributions}

All authors contributed equally to the writing of this paper. All authors read and approved the final manuscript.

\section{References}

[1] C. Garodia, I. Uddin, A new iterative method for solving split feasibility problem, J. Appl. Anal. Comput., 10(3) (2020), 986-1004.

[2] C. Garodia, I. Uddin, A new fixed point algorithm for finding the solution of a delay differential equation, AIMS Math., 5 (4) (2020), 3182-3200.

[3] E. Hacıoğlu, F. Gürsoy, S. Maldar, Y. Atalan, G. V. Milovanović, Iterative approximation of fixed points and applications to two-point second-order boundary value problems and to machine learning, Appl. Numer. Math., 167 (2021), 143-172.


mappings, J. Appl. Math. Comput., (2021). https://doi.org/10.1007/s12190-021-01552-7.

[5] S. Maldar, Y. Atalan, K. Doğan, Comparison rate of convergence and data dependence for a new iteration method, Tbilisi Math. J., 13(4) (2020), $65-79$.

[6] S. Maldar, An examination of data dependence for Jungck-type iteration method, Erciyes Univ. J. Inst. Sci. Tech., 36 (3) (2020), $374-384$.

[7] E. Hacioğlu, V. Karakaya, Existence and convergence for a new multivalued hybrid mapping in CAT( $\kappa)$ spaces, Carpathian J. Math., 33(3) (2017), 319-326.

[8] E. Hacioğlu, V. Karakaya, Some fixed point results for a multivalued generalization of generalized hybrid mappings in CAT( $\kappa)$-spaces, Konuralp J. Math., 6(1) (2018), 26-34.

[9] E. Hacioğlu, V. Karakaya, A new contraction-like multivalued mapping on geodesic spaces, Sci. Stud. Res. Ser. Math. Inform., 29(1) (2019), 89-102.

[10] F. Gürsoy, K. Doğan, A. R. Khan, Direct estimate of accumulated errors for a general iteration method, Math. Adv. Pure Appl. Sci. (MAPAS), 2(2019),

[11] Y. Xu, Z. Liu, On estimation and control of errors of the Mann iteration process, J. Math. Anal. Appl., 286 (2003), $804-806$.

[12] Y. Xu, Z. Liu, S. M. Kang, Accumulation and control of random errors in the Ishikawa iterative process in arbitrary Banach space, Comput. Math. Appl., 61 (2011), 2217-2220.

[13] S. Thianwan, S. Suantai, Convergence criteria of a new three-step iteration with errors for nonexpansive nonself-mappings, Comput. Math. Appl., 52 (2006), 1107-1118.

[14] K. Nammanee, S. Suantai, The modified Noor iterations with errors for non-Lipschitzian mappings in Banach spaces, Appl. Math. Comput., 187 (2007), 669-679.

[15] K. Nammanee, M. A. Noor, S. Suantai, Convergence criteria of modified Noor iterations with errors for asymptotically nonexpansive mappings, J. Math. Anal. Appl., 314 (2006), 320-334.

[16] M. A. Noor, New approximation schemes for general variational inequalities, J. Math. Anal. Appl., 251 (2000) $217-229$.

[17] S. Ishikawa, Fixed points by a new iteration method, Proc. Amer. Math. Soc., 44 (1974), 147-150.

[18] W. R. Mann, Mean value methods in iteration, Proc. Amer. Math. Soc., 4 (1953), 506-510.

[19] S. M. Şoltuz, T. Grosan, Data dependence for Ishikawa iteration when dealing with contractive-like operators, Fixed Point Theory A., 2008 (2008), $1-7$. 